\title{
A Proactive Maintenance Scheme for Hardware Fault Diagnosis in Wireless Systems
}

\author{
Barbara Walsh ${ }^{\dagger}$ and Ronan Farrell* \\ Centre for Telecommunications \\ Value-Chain Research \\ Department of Electronic Engineering \\ National University of Ireland, Maynooth \\ E-mail: †bwalsh@eeng. nuim.ie \\ Institute of Microelectronics \\ and Wireless Systems \\ Department of Electronic Engineering \\ National University of Ireland, Maynooth \\ *rfarrelldeeng.nuim.ie
}

\begin{abstract}
In the wireless sector maintenance costs form a large part of the total network operating cost. This paper presents a possible proactive maintenance scheme for wireless systems. The object of this paper is to reduce the high operational costs encountered in the wireless industry by decreasing maintenance costs and system downtime. An on-line monitoring system is suggested to identify performance degradation, as well as its possible sources via symbol frequency distribution analysis. In this paper a single fault mechanism is considered and the methodology to detect and diagnose the fault mechanism is presented. With similar analysis for other fault mechanisms, this system could ensure that maintenance occurs only when necessary and not at routine intervals.
\end{abstract}

Keywords - RF Hardware, Fault Detection, Fault Diagnosis

\section{INTRODUCTION}

Modern communications systems are becoming increasingly complex so as to meet the burgeoning requirements of consumer applications. The fees at which these services are provided are highly competitive. To maximise profits while meeting requirements such as coverage, capacity and quality of service network operators must take into account financial as well as wireless constraints.

The many costs incurred while setting up and running a wireless network may be divided into two broad categories: capital expenditure (CAPEX) and operational expenditure (OPEX). CAPEX includes investments made in the networks such, as base station and antenna installation costs, as well as the hardware necessary to carry out operation administration, maintenance and provisioning (OAM\&P) functions such as billing systems [1]. OPEX consists of operational, administrative, provisioning and maintenance costs of the network [1].

As a consequence of the competitive environment and high OPEX, it is essential that all systems are as efficient as possible and that unnecessary expenditure is cut. Service providers require equipment that is cheap and easy to install, uses little power and seldom requires maintenance [2].

Within these intricate systems there are many possible sources of failure. The RF hardware in particular is quite prone to failure due to the challenging thermal conditions and high power loads to which they are subjected.

When failure occurs, the cost for wireless service providers is extensive both in terms of lost revenue and diminishing customer confidence. In an attempt to reduce the duration of service interruption, considerable measures are taken by the service providers. The principal measure being frequent and often unnecessary preventative maintenance of base stations and antennas. An example of these high network maintenance costs can be seen in Vodafone's published figures [3] for worldwide operational costs. With a total revenue of $£ 14.829$ billion, the total network operating cost is stated as $£ 8.407$ billion, where approximately $50 \%$ of these costs can be attributed to network upkeep.

Elevated maintenance costs are compounded by the lack of standard diagnostics within mobile base stations. Many base station manufacturers make use of 
third party vendor sub-systems and components when constructing base stations. Often these sub-systems lack self test capabilities or have proprietary diagnostic interfaces. These diagnostic interfaces often provide much information about the status of the system which is neither well understood nor used in a productive manner resulting in generally poor base station diagnostic capabilities. A considerable burden is placed on the engineering resources of the operator by this multitude of proprietary interfaces which is beyond the capabilities of all but the largest. It is not uncommon within the wireless industry for a fault to be diagnosed only after service has been interrupted. This inefficiency in terms of diagnostics and hence maintenance, results in unnecessarily high expenditure on the part of the service provider to maintain a level of service which is acceptable to the consumer.

As competition within the wireless communications sector is constantly escalating, both in terms of services offered and fees charged, the reduction of network operation and maintenance costs is extremely desirable. To this end a proactive maintenance scheme for hardware fault detection in wireless networks which would result in reduced service interruption is proposed.

\section{a) Proposed Solution}

In this paper an on-line monitoring system with low operational overhead which would monitor the system operation is proposed. Any change in performance, and in particular any degradation in performance, would be logged and its cause diagnosed. This performance degeneration diagnosis would be achieved through the analysis of the symbol frequency distribution of the received signal of the communications system. Hence, it becomes possible not only to detect and diagnose the cause of failure or degradation before reaching the base station, but to minimise the amount of time spent on site carrying out repairs. Furthermore, failure of components and sub-systems can be predicted by monitoring any decline in performance, ensuring that maintenance occurs before service interruption.

The power amplification process in particular is prone to failure. Failure of the power amplifier (PA) results in failure of the system. Hence, the PA shall be used as the test case for the proactive maintenance scheme described in this paper. In the future other hardware faults and operational environments will be considered.

The paper is organized as follows: Section II gives an overview of the proposed proactive maintenance system. In Section III the theoretical framework for the fault diagnosis tool is provided. Results are presented in Section IV while Section V describes the software implementation of the diagnosis mechanism and Section VI sets forth the conclusions.

\section{SySTEM OVERVIEW}

A block diagram of the proactive maintenance system advocated in this paper can be seen in Fig. 2 .

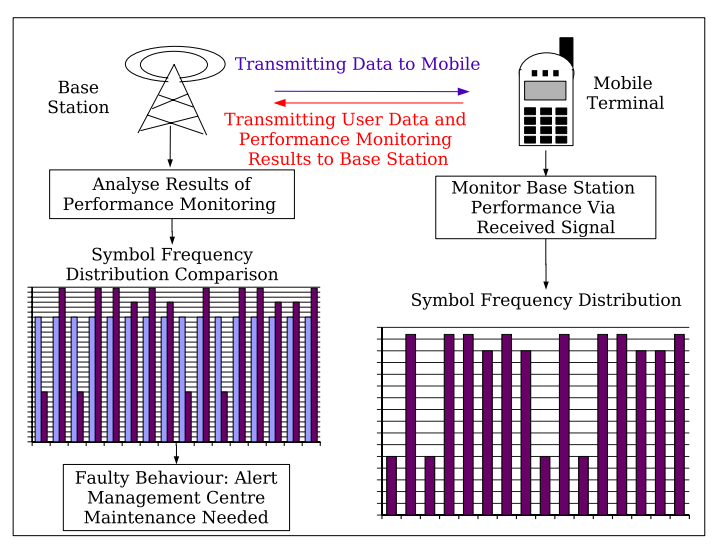

Fig. 1: Proactive Maintenance System Diagram

The algorithm used by the proposed proactive maintenance scheme ensures that maintenance occurs only when strictly necessary. It is based on symbol frequency distributions which are calculated and used as follows:

- Base station transmits data to the mobile terminal.

- Mobile terminal counts the number of times each of the $M$ constellation symbol is received, $\# S_{i}$, and the total number of symbols received, $S_{R X}$.

- $\# S_{i}$ and $S_{R X}$ are transmitted to the base station by the mobile terminal at regular intervals.

- The base station calculates the symbol frequency of each of the $M$ constellation symbol as follows:

$F_{S}=\frac{\# S_{i}}{S_{R X}}$

- This symbol frequency distribution, $F_{S}$, is compared to the expected distribution for correct operation, $F_{E X}$.

- If the distributions do not match then the distribution, $F_{S}$, is compared to a set of possible distributions, $F_{K N O W N}$, which represent certain error mechanisms.

- The network management centre is alerted that maintenance is needed, with a diagnosis if one is available.

- If the symbol frequency distribution, $F_{S}$, matches the expected distribution, $F_{E X}$, continue to monitor the system. 


\section{THEORETICAL FRAMEWORK}

As previously mentioned, the system performance monitoring is carried out via the symbol frequency distribution of the received signal. These distributions are verified theoretically via the error probability of each of the $\mathrm{M}$ constellation symbols. This is briefly discussed in this section. A more in depth analysis of this theory may be found in [4].

It is proposed that the error probability of each individual symbol is effected by nonlinear distortion and that these error probabilities have a distinctive pattern associated with a particular form of distortion. In this section this theory is outlined for the error probability of a PA with gain compression, which is the proactive maintenance system test case. The error probabilities for M-ary Quadrature Amplitude Modulation (MQAM) with an additive white Gaussian noise (AWGN) channel and M-QAM with a failing PA and an AWGN channel are given.

The probability of symbol error, $P_{M}$, for M-QAM with an AWGN channel is given by [5]:

$P_{M}=1-\left(1-P_{L}\right)^{2}$

where $P_{L}$ is the error probability of L-ary Pulse Amplitude Modulation (L-PAM) and is given by [5]:

$P_{L}=2\left(1-\frac{1}{L}\right) Q\left(\sqrt{\frac{3}{L^{2}-1} \frac{E_{S}}{N_{O}}}\right)$

where $E_{S} / N_{O}$ is the signal to noise ratio (SNR) of the L-PAM signal and $L=\sqrt{M}$.

The error probability of each individual M-QAM symbol, as opposed to the overall probability of symbol error, can be calculated numerically using the method proposed by Craig in [6] which may be summarized as follows.

If an arbitrary M-QAM constellation symbol, $S_{N}$, is transmitted, an error in reception occurs if the noise causes the receiver to produce a point outside the decision boundaries B1, B2, B3 or B4 as shown in Fig. 2. Otherwise it is received correctly. In the AWGN case, each of the sub-sections of the decision region shown in Fig. 2 are identical since ideally, the symbol is transmitted at the exact centre of the decision region. The probability that detected point lies outside a boundary is given by [6]:

$P_{S_{N}}=\frac{1}{\pi} \sum_{k=1}^{n} \int_{0}^{\theta_{k}} \exp \left[\frac{-X_{k}^{2} \sin ^{2}\left(\Psi_{k}\right)}{2 \sigma^{2} \sin ^{2}\left(\theta+\Psi_{k}\right)}\right] d \theta$

where $\mathrm{k}$ is the number of decision regions surrounding the the constellation symbol. For any signal point, the number of terms in the sum, $P_{S_{N}}$, will be equal to the number of sides on its decision region.

The error probability including PA nonlinearity cannot be found using standard error probability formulas such as equation (2) and so it is also calculated using

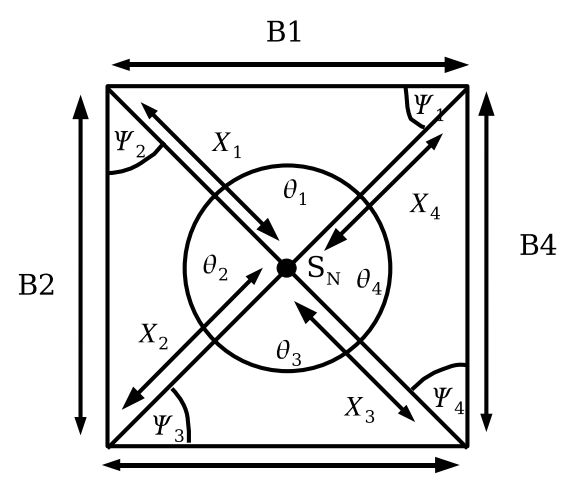

B3

Fig. 2: Signal Point and its Decision Boundaries for M-QAM with AWGN Channel

the methodology proposed by [6]. However, the decision region is divided into different sub-sections than in the AWGN case. When the symbol is passed through a nonlinear PA before transmission, compression occurs, which results in the symbol moving away from the centre of the decision region. An example of an arbitrary M-QAM constellation symbol distorted by a nonlinear PA is shown in Fig. 3

B1

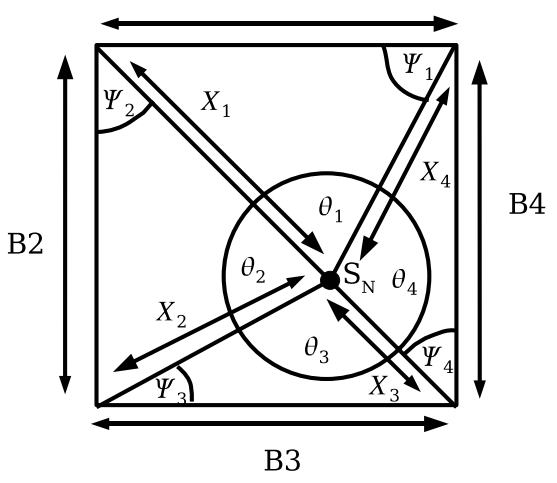

Fig. 3: Signal Point and its Decision Boundaries for M-QAM with a Nonlinear PA and AWGN Channel

The distortion visible in Fig. 3 will cause a variation in the receive probabilities for each constellation symbol. The variation in the receive probabilities, and the manner in which symbols are incorrectly received, is characteristic of the various failure modes, allowing for both detection and diagnosis.

In the next section, some results for the test case, an aging power amplifier within degraded linearity performance are presented.

\section{RESUlTS}

System performance monitoring was simulated in Matlab for a 16-QAM signal with an AWGN channel and a 16-QAM signal with a failing PA and an AWGN channel. The failing PA is modeled as an aging amplifier 
with degraded linearity performance using the Rapp model. The AM-to-AM and AM-to-PM conversions were applied according to the following functions [7]:

$$
\begin{aligned}
F_{A M / A M} & =\frac{u}{\left[1+\left(\frac{u}{V_{S A T}}\right)^{2 p}\right]^{\frac{1}{2 p}}} \\
F_{A M / P M} & =0
\end{aligned}
$$

where $\mathrm{u}$ is the magnitude of the input signal, $\mathrm{p}$ is the smoothness factor and $V_{S A T}$ is the maximum output amplitude.

Fig. 4 clearly shows that the simulated and calculated results in terms of bit error rate (BER) are closely matched for a 16-QAM signal with an AWGN channel and a 16-QAM signal with a nonlinear PA $\left(p=3, V_{S A T}=3 V, 3.5 V\right)$ and an AWGN channel. They simulations were carried out over a range of $E_{B} / N_{O}$ from $0 d B$ to $20 d B$.

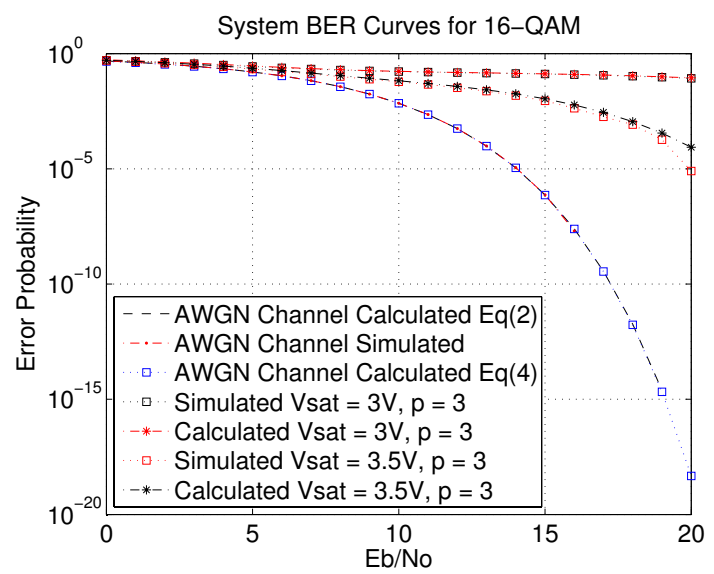

Fig. 4: BER curve for 16-QAM with AWGN Channel and 16-QAM with AWGN Channel and Nonlinear PA

Fig. 5, Fig. 6 and Fig. 7 show the simulated and calculated probability of error for each individual constellation symbol in three separate simulation environments: a 16-QAM signal with AWGN channel, a 16QAM signal with AWGN channel and nonlinear PA with parameters $p=3$ and $V_{S A T}=3 \mathrm{~V}, 3.5 \mathrm{~V} . E_{B} / N_{O}$ is set to $12 d B$ in each case. As can be seen in Fig. 5, Fig. 6 and Fig. 7 there is a strong similarity between the simulated and calculated results.

Having authenticated the simulation results, the symbol frequency distribution which is the performance monitoring mechanism of the proactive maintenance scheme is now considered. The symbol frequency of a 16-QAM signal with an AWGN channel and a 16-QAM signal with an AWGN channel and a PA with different levels of degraded linearity performance is shown in Fig. 8. These symbol frequencies were calculated using Equation (1).

There is an obvious pattern here. When considering the AWGN channel each symbol has the same frequency of occurrence, $F_{S, 16-Q A M}=1 / 16$, which is

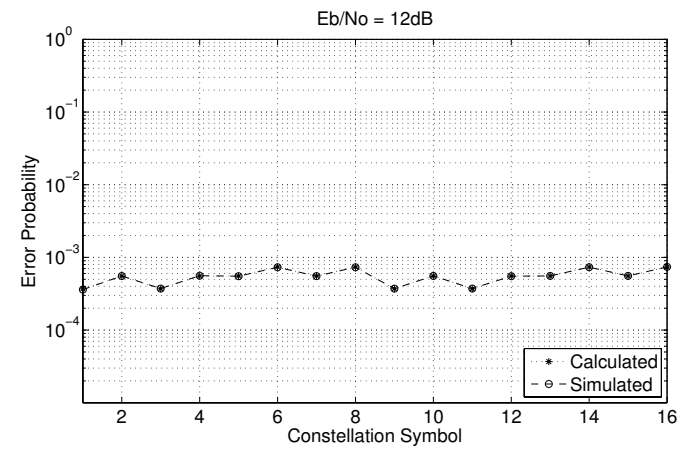

Fig. 5: Individual Symbol Error Probability for 16-QAM with AWGN Channel

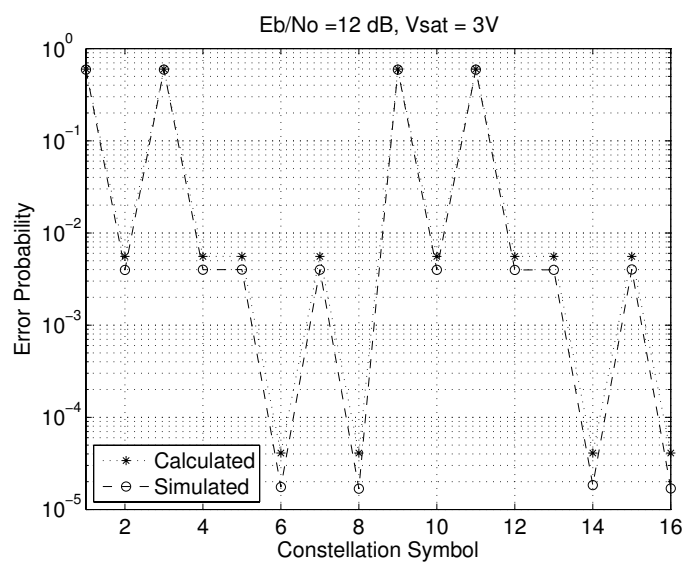

Fig. 6: Individual Symbol Error Probability for 16-QAM with AWGN Channel and Nonlinear PA, $V_{S A T}=3 \mathrm{~V}$

independent of $E_{B} / N_{O}$. The 16-QAM signal with a nonlinear PA and an AWGN channel simulation yields the relative frequency plots which can also be seen in Fig. 8. In this instance there is also a very clear pattern which is followed over the range of $E_{B} / N_{O}$. The symbols fall into three distinct categories depending on the level of compression to which they are subjected. Moreover, the value of the symbol frequency is affected by the level of nonlinearity and hence can be used to detect a change in the PA performance over time. When the symbol frequency distribution for a 16QAM signal with an AWGN channel is contrasted with a 16-QAM signal with a nonlinear PA and an AWGN channel the resulting distributions are distinctively dissimilar. Ergo, the source of degradation can be identified as gain compression with great facility on this occasion.

The proactive maintenance system was simulated for a range of values of $E_{B} / N_{O}$ from $0 d B$ to $20 d B$ with degraded linearity. Its overall performance for $V_{S A T}=3 V, 3.5 \mathrm{~V}$ and $p=3$ can be seen in Fig. 9 . This performance measure is based on the Euclidean distance, $D$, between the maximum symbol frequency, $F_{S_{M A X}}$, and minimum symbol frequency, $F_{S_{M I N}}$, for each value of $E_{B} / N_{O}$. It can be stated as follows:

$D=\left|F_{S_{M A X}}-F_{S_{M I N}}\right|$ 


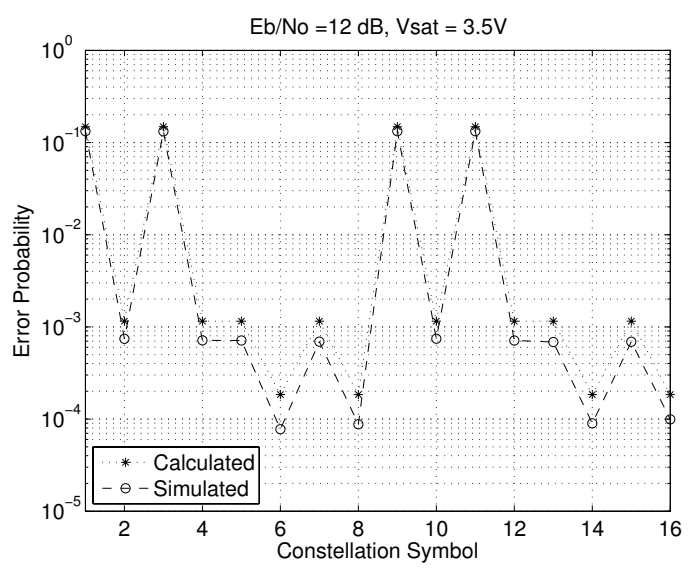

Fig. 7: Individual Symbol Error Probability for 16-QAM with AWGN Channel and Nonlinear PA, $V_{S A T}=3.5 \mathrm{~V}$

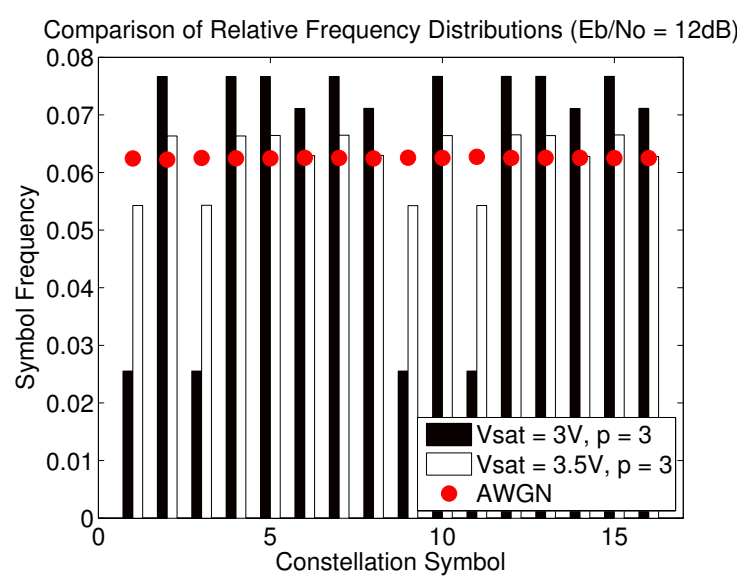

Fig. 8: Comparison of Symbol Frequency for Each Constellation Point for 16-QAM with AWGN Channel and 16-QAM with Nonlinear PA and AWGN channel

As can be clearly seen, there is a distinct difference between the levels of the symbol frequency which makes the distortion pattern pronounced. As a result the source of performance degradation can be determined. Furthermore, the worse the linearity performance of the PA, the more noticeable the characteristic pattern in the symbol frequency distribution becomes.

\section{SOFTWARE IMPLEMENTATION OF FAULT DiAGNOSIS MECHANISM}

The software implementation of the fault diagnosis mechanism is carried out via the use of classification rules.

The diagnostic problem may be stated as follows. The system, $\mathrm{S}$, is constructed from a set of components $C=\left(C_{1}, C_{2}, \ldots, C_{N}\right)$ [8]. The performance of the system is monitored periodically using a set of tests $T=\left(T_{1}, T_{2}, \ldots, T_{M}\right)$ to determine whether or not the system is operating as expected [8]. If it is not, $\mathrm{T}$ is then used to determine which of the $\mathrm{C}$ components have caused the performance degradation of $\mathrm{S}$. The attribute which is tested to establish the system status is the sym-

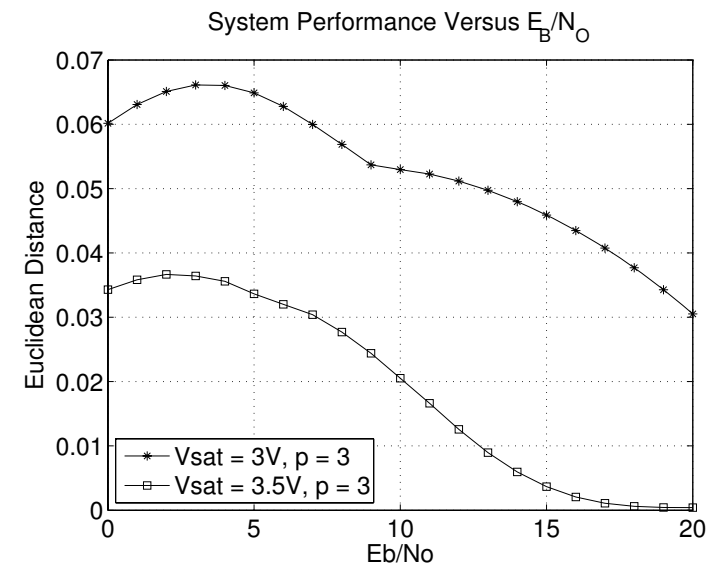

Fig. 9: Performance of Proactive Maintenance System

\section{bol frequency.}

The testing sequence may be seen in Fig. 10. Firstly, the system performance is compared that the reference symbol frequency distribution to ascertain if system performance is as expected. If not the system symbol frequency distribution is then compared to other known fault mechanisms to discover the source of performance degradation.

The system was simulated for a range of values of $V_{S A T}, p$ and $E_{B} / N_{O}$ with correct classification of the faults over the range of parameter values. $100 \%$ of symbol distributions were correctly classified by the fault diagnosis mechanism as either being caused by an AM-to-AM conversion introduced by the PA, by an AWGN channel (which is considered to be the ideal system operation) or by some other, as yet unknown, fault.

Furthermore a set of random symbol frequency distributions was generated. The confidence factor with which the diagnosis is made is defined as [9]:

Confidence $=\frac{|A \cap y|}{|A|}$

where $|A|$ is the number of records which satisfy the classification rule and $|A \cap y|$ is the number of records which satisfy both the rule antecedent (or precondition) and the rule consequent (or classification). The proactive maintenance system presented in this paper has a confidence factor of $99.8 \%$ when a set of random symbol frequency distributions were introduced. Hence, the system makes the correct fault diagnosis for the test case when presented with the ideal behaviour, a known fault (a PA with AM-to-AM conversion) and unknown faults.

\section{CONCLUSIONS}

Growing user requirements and market competition in the wireless sector intensify the need for reduced operational expenditure by wireless service providers. With this aim in mind a scheme which would reduce maintenance costs and system downtime has been presented. 


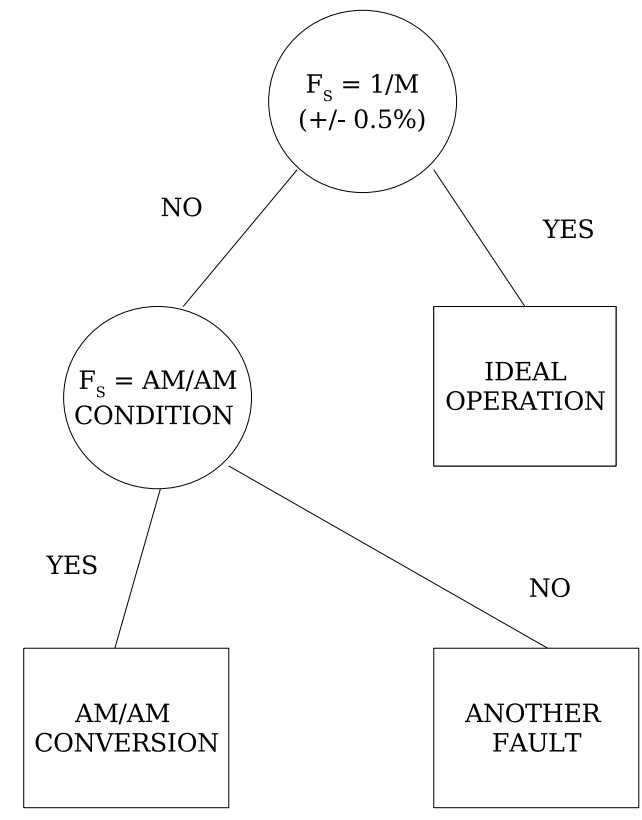

Fig. 10: Fault Diagnosis Test Sequence

This system is based on a simple algorithm with low overhead which could be easily implemented. It has been shown that this scheme, based on symbol frequency distributions, can be used to identify hardware faults using a PA as the test case. The symbol frequency distribution of the failing PA is easily differentiable from the symbol frequency distribution of an AWGN channel.

Generation and verification of symbol frequency distributions for other faults and operational environments will be carried out in the future.

\section{ACKNOWLEDGMENTS}

The authors would like to thank the Centre for Telecommunications Value-Chain Research (CTVR) and the Science Foundation of Ireland (SFI) for supporting this research.

\section{REFERENCES}

[1] T. Smura. "Techno-economic analysis of 802.16a-based fixed wireless access networks". Master's thesis, Helsinki University of Technology, 2004.

[2] D. Jones. "Base stations drive wireless economics" http://www.unstrung.com/, May 20, 2008.
[3] A. Sarin. "Vodafone group plc interim results for the six months to 30 september 2004". http://www.vodafone.com/, May 20, 2008.

[4] B. Walsh, R. Farrell, and G. Baldwin. "Physical Component Performance Degradation Detection Based on Error Probability Analysis". Proceedings of the 18th Annual IEEE International Symposium on Personal, Indoor and Mobile Radio Communications, 2007.

[5] J. Proakis. Digital Communications, McGraw Hill, 2001.

[6] J. Craig. "A new, simple exact result for calculating the probability of error for two-dimensional signal constellations". Proceedings of the IEEE Military Communications Conference, vol. 2, pages II571 - II575, 1991.

[7] C. Rapp. "Effects of HPA-Nonlinearity on a 4DPSK/OFDM-Signal for a Digital Sound Broadcasting System". Proceedings of the Second European Conference on Satellite Communications, pp. 179-184, 1991.

[8] T. Assaf and J.B. Dugan. "Diagnosis based on reliability Analysis Using Monitors and Sensors". Reliability Engineering and System Safety, vol. 93, pages 509 - 521, 2008.

[9] P.N. Tan, M. Steinbach and V. Kumar. Introduction to Data Mining Addison Wesley, 2006. 